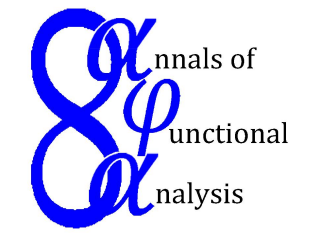

Ann. Funct. Anal. 5 (2014), no. 2, 147-157

$\mathscr{A}$ NNALS OF $\mathscr{F}$ UNCTIONAL $\mathscr{A}$ NALYSIS

ISSN: 2008-8752 (electronic)

URL:www.emis.de/journals/AFA/

\title{
ON $f$-CONNECTIONS OF POSITIVE DEFINITE MATRICES
}

\author{
MAREK NIEZGODA \\ This paper is dedicated to Professor Tsuyoshi Ando
}

Communicated by K. S. Berenhaut

\begin{abstract}
In this paper, by using Mond-Pečarić method we provide some inequalities for connections of positive definite matrices. Next, we discuss specifications of the obtained results for some special cases. In doing so, we use $\alpha$-arithmetic, $\alpha$-geometric and $\alpha$-harmonic operator means.
\end{abstract}

\section{Introduction}

Throughout $M_{n}(\mathbb{C})$ denotes the $C^{*}$-algebra of $n \times n$ complex matrices. For matrices $X, Y \in \mathbb{M}_{n}(\mathbb{C})$, the notation $Y \leq X$ (resp., $\left.Y<X\right)$ means that $X-Y$ is positive semidefinite (resp., positive definite). A linear map $\Phi: \mathbb{M}_{n}(\mathbb{C}) \rightarrow \mathbb{M}_{k}(\mathbb{C})$ is said to be positive if $0 \leq \Phi(X)$ for $0 \leq X \in \mathbb{M}_{n}(\mathbb{C})$. If in addition $0<\Phi(X)$ for $0<X \in \mathbb{M}_{n}(\mathbb{C})$ then $\Phi$ is said to be strictly positive.

A real function $h: J \rightarrow \mathbb{R}$ defined on interval $J \subset \mathbb{R}$ is called an operator monotone function, if for all Hermitian matrices $A$ and $B$ (of the same order) with spectra in $J$,

$$
A \leq B \text { implies } h(A) \leq h(B)
$$

(see [4, p. 112]).

For $\alpha \in[0,1]$, the $\alpha$-arithmetic mean of $n \times n$ positive definite matrices $A$ and $B$ is defined as follows

$$
A \nabla_{\alpha} B=(1-\alpha) A+\alpha B .
$$

For $\alpha=\frac{1}{2}$ one obtains the arithmetic mean $A \nabla B=\frac{1}{2}(A+B)$.

Date: Received: November 5, 2013; Accepted: December 6, 2013.

2010 Mathematics Subject Classification. Primary 15A45; Secondary 47A63, 47A64.

Key words and phrases. Positive definite matrix, $\alpha$-arithmetic ( $\alpha$-geometric, $\alpha$-harmonic) operator mean, positive linear map, operator monotone function, $f$-connection. 
For $\alpha \in[0,1]$, the $\alpha$-geometric mean of $n \times n$ positive definite matrices $A$ and $B$ is defined by

$$
A \sharp_{\alpha} B=A^{1 / 2}\left(A^{-1 / 2} B A^{-1 / 2}\right)^{\alpha} A^{1 / 2}
$$

(see $[9,15])$. In particular, for $\alpha=\frac{1}{2}$ equation (1.2) defines the geometric mean of $A$ and $B$ defined by

$$
A \sharp B=A^{1 / 2}\left(A^{-1 / 2} B A^{-1 / 2}\right)^{1 / 2} A^{1 / 2}
$$

(see $[2,10,15])$.

For $\alpha \in[0,1]$, the $\alpha$-harmonic mean of $n \times n$ positive definite matrices $A$ and $B$ is defined by

$$
A !_{\alpha} B=\left((1-\alpha) A^{-1}+\alpha B^{-1}\right)^{-1} .
$$

For $\alpha=\frac{1}{2}$ we obtain the harmonic mean of $A$ and $B$ given by

$$
A ! B=\left(\frac{1}{2} A^{-1}+\frac{1}{2} B^{-1}\right)^{-1}
$$

(see [11]).

Ando's inequality [1] asserts that if $\Phi: \mathbb{M}_{n}(\mathbb{C}) \rightarrow \mathbb{M}_{k}(\mathbb{C})$ is a positive linear map and $A, B \in \mathbb{M}_{n}(\mathbb{C})$ are positive definite then

$$
\Phi\left(A \sharp_{\alpha} B\right) \leq \Phi(A) \sharp_{\alpha} \Phi(B) .
$$

Lee [10] established the following reverse of inequality (1.4) with $\alpha=\frac{1}{2}$ (see also [12]).

Theorem A [10, Theorem 4] Let $A$ and $B$ be $n \times n$ positive definite matrices. Assume $\Phi: \mathbb{M}_{n}(\mathbb{C}) \rightarrow \mathbb{M}_{k}(\mathbb{C})$ is a positive linear map.

If $m A \leq B \leq M A$ with positive scalars $m, M$ then

$$
\Phi(A) \sharp \Phi(B) \leq \frac{\sqrt{M}+\sqrt{m}}{2 \sqrt[4]{m M}} \Phi(A \sharp B) .
$$

Recently, Seo [15] showed difference and ratio type reverses of Ando's inequality (1.4), as follows.

Theorem B [15, Theorem 1] Let $A$ and $B$ be $n \times n$ positive definite matrices such that $m A \leq B \leq M A$ for some scalars $0<m<M$ and let $\Phi: \mathbb{M}_{n}(\mathbb{C}) \rightarrow$ $\mathbb{M}_{k}(\mathbb{C})$ be a positive linear map.

Then for each $\alpha \in(0,1)$

$$
\Phi(A) \sharp_{\alpha} \Phi(B)-\Phi\left(A \sharp_{\alpha} B\right) \leq-C(m, M, \alpha) \Phi(A),
$$

where the Kantorovich constant for the difference $C(m, M, \alpha)$ is defined by

$$
C(m, M, \alpha)=(\alpha-1)\left(\frac{M^{\alpha}-m^{\alpha}}{\alpha(M-m)}\right)^{\frac{\alpha}{\alpha-1}}+\frac{M m^{\alpha}-m M^{\alpha}}{M-m} .
$$

Theorem C [15, Theorem 3] Let $A$ and $B$ be $n \times n$ positive definite matrices such that $m A \leq B \leq M A$ for some scalars $0<m<M$ and let $\Phi: \mathbb{M}_{n}(\mathbb{C}) \rightarrow$ $\mathbb{M}_{k}(\mathbb{C})$ be a positive linear map.

Then for each $\alpha \in(0,1)$

$$
\Phi(A) \sharp_{\alpha} \Phi(B) \leq K(m, M, \alpha)^{-1} \Phi\left(A \sharp_{\alpha} B\right),
$$


where the generalized Kantorovich constant $K(m, M, \alpha)$ is defined by

$$
K(m, M, \alpha)=\frac{m M^{\alpha}-M m^{\alpha}}{(\alpha-1)(M-m)}\left(\frac{\alpha-1}{\alpha} \frac{M^{\alpha}-m^{\alpha}}{m M^{\alpha}-M m^{\alpha}}\right)^{\alpha} .
$$

Theorem D [8, Theorem 2.1] Let $A$ and $B$ be $n \times n$ positive definite matrices such that $0<b_{1} \leq A \leq a_{1}$ and $0<b_{2} \leq B \leq a_{2}$ for some scalars $0<b_{i}<a_{i}$, $i=1,2$.

If $\Phi: \mathbb{M}_{n}(\mathbb{C}) \rightarrow \mathbb{M}_{k}(\mathbb{C})$ is a strictly positive linear map, then for any operator mean $\sigma$ with the representing function $f$, the following double inequality holds:

$$
\omega^{1-\alpha}\left(\Phi(A) \sharp_{\alpha} \Phi(B)\right) \leq(\omega \Phi(A)) \nabla_{\alpha} \Phi(B) \leq \frac{\alpha}{\mu} \Phi(A \sigma B),
$$

where $\mu=\frac{a_{1} b_{1}\left(f\left(b_{2} a_{1}^{-1}\right)-f\left(a_{2} b_{1}^{-1}\right)\right)}{b_{1} b_{2}-a_{1} a_{2}}, \nu=\frac{a_{1} a_{2} f\left(b_{2} a_{1}^{-1}\right)-b_{1} b_{2} f\left(a_{2} b_{1}^{-1}\right)}{a_{1} a_{2}-b_{1} b_{2}}, \omega=\frac{\alpha \nu}{(1-\alpha) \mu}$ and $\alpha \in$ $(0,1)$.

The purpose of this paper is to demonstrate a unified framework including Theorems A, B, C and D as special cases. Following the idea of Mond-Pečarić method [5, 11], in our approach we use a connection $\sigma_{f}$ induced by a continuous function $f: J \rightarrow \mathbb{R}$. We focus on double inequalities as in (1.5) (cf. [6, Theorem 3.1]).

In Section 2, we formulate conditions for four functions $f_{1}, f_{2}, g_{1}, g_{2}$, under which the following double inequality holds (see Theorem 2.3):

$$
c_{g_{2}} \Phi(A) \sigma_{f_{2}} \Phi(B) \leq \Phi\left(A \sigma_{g_{2}} B\right) \leq \Phi\left(A \sigma_{g_{2} g_{1}^{-1}}\left(A \sigma_{f_{1}} B\right)\right),
$$

with suitable constant $c_{g_{2}}$ (see (2.8)). Here the crucial key is the behaviour of the superposition $g_{2} g_{1}^{-1}$. By substituting $\alpha t+1-\alpha, t^{\alpha}$ and $\left(\alpha t^{-1}+1-\alpha\right)^{-1}$ in place of $g_{2} g_{1}^{-1}(t)$, we get variants of the above double inequality (1.6) for $\alpha$-arithmetic, $\alpha$-geometric and $\alpha$-harmonic operator means, respectively. Also, some further substitutions for $f_{1}, f_{2}, g_{2}$ are possible. Thus we can obtain some old and new results as special cases of (1.6) (see Theorem 2.9 and Corollaries 2.6-2.18).

\section{Results}

Let $f: J \rightarrow \mathbb{R}$ be a continuous function on an interval $J \subset \mathbb{R}$. The $f$-connection of an $n \times n$ positive definite matrix $A$ and an $n \times n$ hermitian matrix $B$ such that the spectrum $\operatorname{Sp}\left(A^{-1 / 2} B A^{-1 / 2}\right) \subset J$, is defined by

$$
A \sigma_{f} B=A^{1 / 2} f\left(A^{-1 / 2} B A^{-1 / 2}\right) A^{1 / 2}
$$

(cf. [7, p. 637], [9]).

Note that the operator means (1.1), (1.2) and (1.3) are of the form (2.1) with the functions $\alpha t+1-\alpha, t^{\alpha}$ and $\left(\alpha t^{-1}+1-\alpha\right)^{-1}$, respectively.

For a function $f: J \rightarrow \mathbb{R}_{+}$defined on an interval $J=[m, M]$ with $m<M$, we define

$$
a_{f}=\frac{f(M)-f(m)}{M-m} \quad, \quad b_{f}=\frac{M f(m)-m f(M)}{M-m} \quad \text { and } \quad c_{f}=\min _{t \in J} \frac{a_{f} t+b_{f}}{f(t)}
$$

$($ see $[11])$. 
Lemma 2.1. (See [7, Theorem 1], cf. also [11, Corollary 3.4].) Let $A$ and $B$ be $n \times n$ positive definite matrices such that $m A \leq B \leq M A$ with $0<m<M$.

If $\sigma_{f}$ is a connection with operator monotone concave function $f>0$ and $\Phi$ is a strictly positive linear map, then

$$
c_{f} \Phi(A) \sigma_{f} \Phi(B) \leq \Phi\left(A \sigma_{f} B\right),
$$

where $c_{f}$ is defined by (2.2).

Remark 2.2. (i): For all positive linear maps $\Phi$, the equality

$$
\Phi(A) \sigma_{f} \Phi(B)=\Phi\left(A \sigma_{f} B\right)
$$

holds for the arithmetic operator mean $\sigma_{f}=\nabla_{\alpha}, \alpha \in[0,1]$.

(ii): In general, for other connections $\sigma_{f}$, (2.4) can hold for some specific $\Phi$. For example, taking $\sigma_{f}=\sharp_{\alpha}, \alpha \in[0,1]$, and $\Phi(\cdot)=U^{*}(\cdot) U$ with unitary $U$, we have

$$
U^{*}\left(A \sharp_{\alpha} B\right) U=\left(U^{*} A U\right) \sharp_{\alpha}\left(U^{*} B U\right),
$$

which is of form (2.4).

(iii): Clearly, if the equality (2.4) is met (e.g., if $f$ is affine), then (2.3) holds with $c_{f}=1$ (see (2.20), (2.30)-(2.31)).

Our first result is motivated by [8, Theorem 2.1] (see Theorem D in Section 1).

Theorem 2.3. Let $f_{1}, f_{2}, g_{1}, g_{2}$ be continuous real functions defined on an interval $J=[m, M] \subset \mathbb{R}$. Assume that $g_{2}>0$ and $g_{2} g_{1}^{-1}$ are operator monotone on intervals $J$ and $J^{\prime}=g_{1}(J)$, respectively, with invertible $g_{1}$ and concave $g_{2}$. Let $A$ and $B$ be $n \times n$ positive definite matrices such that $m A \leq B \leq M A$ with $0<m<M$.

If $\Phi: \mathbb{M}_{n}(\mathbb{C}) \rightarrow \mathbb{M}_{k}(\mathbb{C})$ is a strictly positive linear map and

$$
\begin{gathered}
g_{1}(t) \leq f_{1}(t) \text { and } f_{2}(t) \leq g_{2}(t) \quad \text { for } t \in J, \\
\max _{t \in J} g_{1}(t)=\max _{t \in J} f_{1}(t),
\end{gathered}
$$

then

$$
c_{g_{2}} \Phi(A) \sigma_{f_{2}} \Phi(B) \leq \Phi\left(A \sigma_{g_{2}} B\right) \leq \Phi\left(A \sigma_{g_{2} g_{1}^{-1}}\left(A \sigma_{f_{1}} B\right)\right),
$$

where $c_{g_{2}}$ is defined by

$$
a_{g_{2}}=\frac{g_{2}(M)-g_{2}(m)}{M-m} \quad, \quad b_{g_{2}}=\frac{M g_{2}(m)-m g_{2}(M)}{M-m} \quad \text { and } \quad c_{g_{2}}=\min _{t \in J} \frac{a_{g_{2}} t+b_{g_{2}}}{g_{2}(t)} .
$$

Proof. Since $m A \leq B \leq M A$, we obtain $m \Phi(A) \leq \Phi(B) \leq M \Phi(A)$ by the positivity of $\Phi$. In consequence, by the strict positivity of $\Phi$, we get $m \leq W \leq M$ and $\operatorname{Sp}(W) \subset[m, M]$ for $W=\Phi(A)^{-1 / 2} \Phi(B) \Phi(A)^{-1 / 2}$.

It follows from the second inequality of (2.5) that

$$
f_{2}\left((\Phi(A))^{-1 / 2} \Phi(B)(\Phi(A))^{-1 / 2}\right) \leq g_{2}\left((\Phi(A))^{-1 / 2} \Phi(B)(\Phi(A))^{-1 / 2}\right),
$$

and further

$$
\Phi(A) \sigma_{f_{2}} \Phi(B) \leq \Phi(A) \sigma_{g_{2}} \Phi(B) .
$$

According to Lemma 2.1 applied to operator monotone function $g_{2}$, we have

$$
c_{g_{2}} \Phi(A) \sigma_{g_{2}} \Phi(B) \leq \Phi\left(A \sigma_{g_{2}} B\right) .
$$


This and (2.9) imply

$$
c_{g_{2}} \Phi(A) \sigma_{f_{2}} \Phi(B) \leq \Phi\left(A \sigma_{g_{2}} B\right),
$$

proving the left-hand side inequality of (2.7).

It follows that for $h=g_{2} \circ g_{1}^{-1}$,

$$
A \sigma_{g_{2}} B=A \sigma_{h \circ g_{1}} B=A \sigma_{h}\left(A \sigma_{g_{1}} B\right)
$$

where $\circ$ means superposition. In fact, we have

$$
\begin{aligned}
A \sigma_{h \circ g_{1}} B= & A^{1 / 2}\left(h \circ g_{1}\right)\left(A^{-1 / 2} B A^{-1 / 2}\right) A^{1 / 2}=A^{1 / 2} h\left(g_{1}\left(A^{-1 / 2} B A^{-1 / 2}\right)\right) A^{1 / 2} \\
& =A^{1 / 2} h\left(A^{-1 / 2} A^{1 / 2} g_{1}\left(A^{-1 / 2} B A^{-1 / 2}\right) A^{1 / 2} A^{-1 / 2}\right) A^{1 / 2} \\
& =A^{1 / 2} h\left(A^{-1 / 2}\left(A \sigma_{g_{1}} B\right) A^{-1 / 2}\right) A^{1 / 2}=A \sigma_{h}\left(A \sigma_{g_{1}} B\right) .
\end{aligned}
$$

On the other hand, it follows from the first inequality of (2.5) that

$$
g_{1}\left(A^{-1 / 2} B A^{-1 / 2}\right) \leq f_{1}\left(A^{-1 / 2} B A^{-1 / 2}\right)
$$

and next

$$
A \sigma_{g_{1}} B \leq A \sigma_{f_{1}} B
$$

It is seen from (2.5) that

$$
\min _{t \in J} g_{1}(t) \leq \min _{t \in J} f_{1}(t),
$$

which together with (2.6) gives

$$
f_{1}(J) \subset g_{1}(J)
$$

Denote

$$
Z_{0}=A^{-1 / 2}\left(A \sigma_{g_{1}} B\right) A^{-1 / 2}=g_{1}\left(A^{-1 / 2} B A^{-1 / 2}\right)
$$

and

$$
W_{0}=A^{-1 / 2}\left(A \sigma_{f_{1}} B\right) A^{-1 / 2}=f_{1}\left(A^{-1 / 2} B A^{-1 / 2}\right) .
$$

Then $\operatorname{Sp}\left(Z_{0}\right) \subset g_{1}(J)$ and $\operatorname{Sp}\left(W_{0}\right) \subset f_{1}(J)$, because $\operatorname{Sp}\left(A^{-1 / 2} B A^{-1 / 2}\right) \subset J$.

Since $h=g_{2} \circ g_{1}^{-1}$ is operator monotone on $J^{\prime}=g_{1}(J)$, from (2.12) and (2.13) we obtain

and next

$$
h\left(A^{-1 / 2}\left(A \sigma_{g_{1}} B\right) A^{-1 / 2}\right) \leq h\left(A^{-1 / 2}\left(A \sigma_{f_{1}} B\right) A^{-1 / 2}\right)
$$

$$
A \sigma_{h}\left(A \sigma_{g_{1}} B\right) \leq A \sigma_{h}\left(A \sigma_{f_{1}} B\right) .
$$

Therefore, by (2.11) and (2.14), we deduce that

$$
\Phi\left(A \sigma_{g_{2}} B\right) \leq \Phi\left(A \sigma_{g_{2} g_{1}^{-1}}\left(A \sigma_{f_{1}} B\right)\right) .
$$

Now, by combining (2.10) and (2.15), we conclude that (2.7) holds true.

Remark 2.4. In Theorem 2.3, if in addition $f_{1}$ and $g_{1}$ are nondecreasing on $[m, M]$, then condition (2.6) simplifies to

$$
g_{1}(M)=f_{1}(M)
$$

Likewise, if $f_{1}$ and $g_{1}$ are nonincreasing on $[m, M]$, then (2.6) means

$$
g_{1}(m)=f_{1}(m) \text {. }
$$

Corollary 2.5. Under the assumptions of Theorem 2.3. 
(i): If $g_{2} g_{1}^{-1}$ is an affine function, i.e., $g_{2} g_{1}^{-1}(s)=a s+b$ for $s \in g_{1}(J)$, $a>0$, then (2.7) reduces to

$$
c_{g_{2}} \Phi(A) \sigma_{f_{2}} \Phi(B) \leq \Phi\left(A \sigma_{g_{2}} B\right) \leq a \Phi\left(A \sigma_{f_{1}} B\right)+b \Phi(A) .
$$

(ii): If $g_{2} g_{1}^{-1}$ is a power function, i.e., $g_{2} g_{1}^{-1}(s)=s^{\alpha}$ for $s \in g_{1}(J), \alpha \in$ $[0,1]$, then $(2.7)$ reduces to

$$
c_{g_{2}} \Phi(A) \sigma_{f_{2}} \Phi(B) \leq \Phi\left(A \sigma_{g_{2}} B\right) \leq \Phi\left(A \sigma_{\sharp \alpha}\left(A \sigma_{f_{1}} B\right)\right) .
$$

(iii): If $g_{2} g_{1}^{-1}$ is an inverse function of the form $g_{2} g_{1}^{-1}(s)=\left(\alpha s^{-1}+1-\alpha\right)^{-1}$ for $s \in g_{1}(J), \alpha \in[0,1]$, then (2.7) reduces to

$$
c_{g_{2}} \Phi(A) \sigma_{f_{2}} \Phi(B) \leq \Phi\left(A \sigma_{g_{2}} B\right) \leq \Phi\left(\left[(1-\alpha) A^{-1}+\alpha\left(A \sigma_{f_{1}} B\right)^{-1}\right]^{-1}\right) .
$$

Proof. (i). To show (2.16), observe that $a>0$ implies the operator monotonicity of $g_{2} g_{1}^{-1}(s)=a s+b$ (see [4, p. 113]).

It is not hard to verify that

$$
A \sigma_{g_{2} g_{1}^{-1}}\left(A \sigma_{f_{1}} B\right)=a A \sigma_{f_{1}} B+b A .
$$

Hence

$$
\Phi\left(A \sigma_{g_{2} g_{1}^{-1}}\left(A \sigma_{f_{1}} B\right)\right)=a \Phi\left(A \sigma_{f_{1}} B\right)+b \Phi(A) .
$$

Now, it is sufficient to apply (2.7).

(ii). To see (2.17), it is enough to use (2.7) together with the operator monotonicity of $g_{2} g_{1}^{-1}(s)=s^{\alpha}$ with $\alpha \in[0,1]$ (see [4, p. 115]).

(iii). Finally, (2.18) is an easy consequence (2.7) for the operator monotone function $g_{2} g_{1}^{-1}(s)=\left(\alpha s^{-1}+1-\alpha\right)^{-1}$ with $\alpha \in[0,1]$ (see [4, p. 114]).

The next result develops some ideas in $[12,14]$.

Corollary 2.6. Let $f_{1}, f_{2}, g$ be continuous real functions defined on an interval $J=[m, M]$ with invertible operator monotone concave $g>0$ on $J$. Let $A$ and $B$ be $n \times n$ positive definite matrices such that $m A \leq B \leq M A$.

If $\Phi: \mathbb{M}_{n}(\mathbb{C}) \rightarrow \mathbb{M}_{k}(\mathbb{C})$ is a strictly positive linear map and

$$
\begin{gathered}
f_{2}(t) \leq g(t) \leq f_{1}(t) \quad \text { for } t \in J, \\
\max _{t \in J} g(t)=\max _{t \in J} f_{1}(t),
\end{gathered}
$$

then

$$
c_{g} \Phi(A) \sigma_{f_{2}} \Phi(B) \leq \Phi\left(A \sigma_{g} B\right) \leq \Phi\left(A \sigma_{f_{1}} B\right),
$$

where $c_{g}$ is defined by (2.8) for $g_{2}=g$.

In particular, if $g$ is an affine function, i.e., $g(t)=a t+b$ for $t \in J, a>0$, then (2.19) reduces to

$$
\Phi(A) \sigma_{f_{2}} \Phi(B) \leq b \Phi(A)+a \Phi(B) \leq \Phi\left(A \sigma_{f_{1}} B\right) .
$$

Proof. It is enough to apply Theorem 2.3 with $g_{1}=g_{2}=g$. Then the superposition $g_{2} \circ g_{1}^{-1}$ is the identity function $s \rightarrow s, s \in g(J)$. So, (2.16) reads as (2.19).

To see (2.20), use (2.19) with $c_{g}=1$ (see Remark 2.2).

Remark 2.7. The right-hand inequality in (2.20) can be used to obtain DiazMetcalf type inequalities $[8,14]$. 
Remark 2.8. A specialization of Corollary 2.6 leads to [8, Theorem 2.1] (see Theorem $\mathbf{D}$ in Section 1).

Namely, it is easy to verify that the spectrum $\operatorname{Sp}(Z) \subset J$, where $Z=A^{-1 / 2} B A^{-1 / 2}$ and $J=[m, M]$ with $m=\frac{b_{2}}{a_{1}}$ and $M=\frac{a_{2}}{b_{1}}$.

By weighted arithmetic-geometric inequality (see [8])

$$
t^{\alpha} \omega^{1-\alpha} \leq \alpha t+(1-\alpha) \omega \text { for } \alpha \in[0,1] \text { and } t>0, \omega>0 \text {. }
$$

Since $\sigma=\sigma_{f}$ with operator monotone function $f$ on $[0, \infty), f$ must be strictly increasing and concave. Hence

$$
\mu t+\nu \leq f(t) \text { for } t \in J
$$

As a consequence,

$$
\alpha t+(1-\alpha) \omega \leq \frac{\alpha}{\mu} f(t) \quad \text { for } t \in J
$$

By setting

$$
f_{1}(t)=\frac{\alpha}{\mu} f(t), \quad f_{2}(t)=t^{\alpha} \omega^{1-\alpha}, \quad g(t)=(1-\alpha) \omega+\alpha t, \quad t \in J,
$$

we see that conditions (2.5)-(2.6) are satisfied (cf. (2.21)-(2.22) and Remark 2.4). Moreover,

$$
\sigma_{f_{2}}=\sharp_{\alpha} \text { and } \sigma_{g}=\nabla_{\alpha}
$$

Now, it is not hard to check that inequalities (2.20) in Corollary 2.6 applied to the matrices $\omega A$ and $B$ yield (1.5), as required.

The special case of Theorem 2.3 for $f_{1}=f_{2}=f$ gives the following result.

Theorem 2.9. Let $f, g_{1}, g_{2}$ be continuous real functions defined on an interval $J=[m, M]$. Assume $g_{2}>0$ and $g_{2} g_{1}^{-1}$ are operator monotone on $J$ and $J^{\prime}=$ $g_{1}(J)$, respectively, with invertible $g_{1}$ and concave $g_{2}$. Let $A$ and $B$ be $n \times n$ positive definite matrices such that $m A \leq B \leq M A$.

If $\Phi: \mathbb{M}_{n}(\mathbb{C}) \rightarrow \mathbb{M}_{k}(\mathbb{C})$ is a strictly positive linear map and

then

$$
\begin{gathered}
g_{1}(t) \leq f(t) \leq g_{2}(t) \quad \text { for } t \in J, \\
\max _{t \in J} g_{1}(t)=\max _{t \in J} f(t),
\end{gathered}
$$

$$
c_{g_{2}} \Phi(A) \sigma_{f} \Phi(B) \leq \Phi\left(A \sigma_{g_{2}} B\right) \leq \Phi\left(A \sigma_{g_{2} g_{1}^{-1}}\left(A \sigma_{f} B\right)\right)
$$

where $c_{g_{2}}>0$ is given by (2.8).

Proof. Apply Theorem 2.3 for $f_{1}=f_{2}=f$.

Corollary 2.10. Under the assumptions of Theorem 2.9.

(i): If $g_{2} g_{1}^{-1}$ is an affine function, i.e., $g_{2} g_{1}^{-1}(s)=$ as $+b$ for $s \in g_{1}(J)$, $a>0$, then $(2.23)$ reduces to

$$
c_{g_{2}} \Phi(A) \sigma_{f} \Phi(B) \leq \Phi\left(A \sigma_{g_{2}} B\right) \leq a \Phi\left(A \sigma_{f} B\right)+b \Phi(A) .
$$

(ii): If $g_{2} g_{1}^{-1}$ is a power function, i.e., $g_{2} g_{1}^{-1}(s)=s^{\alpha}$ for $s \in g_{1}(J), \alpha \in$ $[0,1]$, then $(2.23)$ reduces to

$$
c_{g_{2}} \Phi(A) \sigma_{f} \Phi(B) \leq \Phi\left(A \sigma_{g_{2}} B\right) \leq \Phi\left(A \sigma_{\sharp \alpha}\left(A \sigma_{f} B\right)\right) .
$$


(iii): If $g_{2} g_{1}^{-1}$ is an inverse function of the form $g_{2} g_{1}^{-1}(s)=\left(\alpha s^{-1}+1-\alpha\right)^{-1}$ for $s \in g_{1}(J), \alpha \in[0,1]$, then (2.23) reduces to

$$
c_{g_{2}} \Phi(A) \sigma_{f} \Phi(B) \leq \Phi\left(A \sigma_{g_{2}} B\right) \leq \Phi\left(\left[(1-\alpha) A^{-1}+\alpha\left(A \sigma_{f_{1}} B\right)^{-1}\right]^{-1}\right) .
$$

Proof. Apply Theorem 2.9.

Remark 2.11. (i): It is worth emphasing that the above inequality (2.24) can be viewed as a reverse inequality of Aujla and Vasudeva [3]:

$$
\Phi\left(A \sigma_{f} B\right) \leq \Phi(A) \sigma_{f} \Phi(B)
$$

for an operator monotone function $f:(0, \infty) \rightarrow(0, \infty)$.

(ii): In the case $f(t)=t^{1 / 2}$ inequality (2.24) is similar to that in [11, Corollary 3.7$]$.

By employing the second part of Theorem 2.9 for some special functions $g_{1}$ and $g_{2}$ we obtain the following.

Corollary 2.12. Let $f: J \rightarrow \mathbb{R}$ and $g: J \rightarrow \mathbb{R}$ be continuous real functions with interval $J=[m, M]$ and invertible operator monotone concave $g$ on $J$. Let $A$ and $B$ be $n \times n$ positive definite matrices such that $m A \leq B \leq M A$.

If $\Phi: \mathbb{M}_{n}(\mathbb{C}) \rightarrow \mathbb{M}_{k}(\mathbb{C})$ is a strictly positive linear map and

$$
\begin{gathered}
a_{1} g(t)+b_{1} \leq f(t) \leq a_{2} g(t)+b_{2} \quad \text { for } t \in J, a_{1}>0, a_{2}>0, \\
\max _{t \in J}\left(a_{1} g(t)+b_{1}\right)=\max _{t \in J} f(t),
\end{gathered}
$$

then

$$
c_{g_{2}} \Phi(A) \sigma_{f} \Phi(B) \leq a_{2} \Phi\left(A \sigma_{g} B\right)+b_{2} \Phi(A) \leq \frac{a_{2}}{a_{1}} \Phi\left(A \sigma_{f} B\right)+\left(b_{2}-\frac{a_{2}}{a_{1}} b_{1}\right) \Phi(A),
$$

where $c_{g_{2}}>0$ is given by (2.8) with $g_{2}=a_{2} g+b_{2}>0$.

If in addition $\operatorname{det}\left(\begin{array}{ll}a_{1} & b_{1} \\ a_{2} & b_{2}\end{array}\right)=0$ then $(2.27)$ becomes

$$
c_{g_{2}} \Phi(A) \sigma_{f} \Phi(B) \leq a_{2} \Phi\left(A \sigma_{g} B\right)+b_{2} \Phi(A) \leq \frac{a_{2}}{a_{1}} \Phi\left(A \sigma_{f} B\right) .
$$

Proof. By putting $g_{1}(t)=a_{1} g(t)+b_{1}$ and $g_{2}(t)=a_{2} g(t)+b_{2}$ for $t \in J$, we find that $g_{2} g_{1}^{-1}: g_{1}(J) \rightarrow \mathbb{R}$ is an affine function, i.e.,

$$
g_{2} g_{1}^{-1}(s)=\frac{a_{2}}{a_{1}} s+b_{2}-\frac{a_{2}}{a_{1}} b_{1} \text { for } s \in g_{1}(J) .
$$

Making use of (2.29) and Theorem 2.9, eq. (2.24), with $a=\frac{a_{2}}{a_{1}}$ and $b=b_{2}-\frac{a_{2}}{a_{1}} b_{1}$ yields (2.27).

Inequality (2.28) is an easy consequence of (2.27).

The special case of Corollary 2.12 for $g(t)=t, t \in J$, leads to some results of Kaur et al. [7, Theorems 1 and 2]. 
Corollary 2.13 (Cf. Kaur et al. [7, Theorems 1 and 2]). Let $f: J \rightarrow \mathbb{R}$ be a continuous real function with interval $J=[m, M]$. Let $A$ and $B$ be $n \times n$ positive definite matrices such that $m A \leq B \leq M A$.

If $\Phi: \mathbb{M}_{n}(\mathbb{C}) \rightarrow \mathbb{M}_{k}(\mathbb{C})$ is a strictly positive linear map, and

$$
\begin{gathered}
a_{1} t+b_{1} \leq f(t) \leq a_{2} t+b_{2} \quad \text { for } t \in J, a_{1}>0, a_{2}>0, \\
a_{1} M+b_{1}=\max _{t \in J} f(t),
\end{gathered}
$$

then

$$
\Phi(A) \sigma_{f} \Phi(B) \leq a_{2} \Phi(B)+b_{2} \Phi(A) \leq \frac{a_{2}}{a_{1}} \Phi\left(A \sigma_{f} B\right)+\left(b_{2}-\frac{a_{2}}{a_{1}} b_{1}\right) \Phi(A) .
$$

If in addition $\operatorname{det}\left(\begin{array}{ll}a_{1} & b_{1} \\ a_{2} & b_{2}\end{array}\right)=0$ then

$$
\Phi(A) \sigma_{f} \Phi(B) \leq a_{2} \Phi(B)+b_{2} \Phi(A) \leq \frac{a_{2}}{a_{1}} \Phi\left(A \sigma_{f} B\right) .
$$

Proof. Use Corollary 2.12, eq. (2.27) and (2.28) with $c_{g_{2}}=1$ (see Remark 2.2).

Remark 2.14. (i): With $a_{1}=a_{2}$, inequality (2.31) can be restated as

$$
\Phi(A) \sigma_{f} \Phi(B) \leq a_{2} \Phi(B)+b_{2} \Phi(A) \leq \Phi\left(A \sigma_{f} B\right) .
$$

This can be obtained for an operator monotone (concave) function $f$ as in the Mond-Pečarić method [5, 11].

(ii): Inequality (2.30) with $a_{1}=a_{2}$ and $f(t)=t^{\alpha}, \sigma_{f}=\sharp_{\alpha}, 0 \leq \alpha \leq 1$, is of type as in Theorem $\mathbf{B}$ (see Section 1).

(iii): When $a_{1} \neq a_{2}$ and $f(t)=t^{\alpha}, \sigma_{f}=\sharp_{\alpha}, 0 \leq \alpha \leq 1$, then (2.31) leads to Theorem C.

(iv): With suitable choosen $a_{1} \neq a_{2}$ and $\sigma_{f}=\sharp_{1 / 2}, f(t)=t^{1 / 2}$, inequality (2.31) can be used to derive Cassels, Kantorovich, Greub-Rheinbold type inequalities, etc. (cf. Theorem A, see also [12, 13, 14] and references therein).

We now consider consequences of Theorem 2.9 for case of geometric mean.

Corollary 2.15. Let $f: J \rightarrow \mathbb{R}$ and $g: J \rightarrow(0,1]$ be continuous real functions with interval $J=[m, M]$ and invertible operator monotone $g$. Let $A$ and $B$ be $n \times n$ positive definite matrices such that $m A \leq B \leq M A$.

If $\Phi: \mathbb{M}_{n}(\mathbb{C}) \rightarrow \mathbb{M}_{k}(\mathbb{C})$ is a strictly positive linear map and, $0<\alpha \leq \beta<1$,

$$
\begin{gathered}
g^{\beta}(t) \leq f(t) \leq g^{\alpha}(t) \quad \text { for } t \in J, \\
\max _{t \in J} g^{\beta}(t)=\max _{t \in J} f(t),
\end{gathered}
$$

then

$$
c_{g_{2}} \Phi(A) \sigma_{f} \Phi(B) \leq \Phi\left(A \sigma_{g^{\alpha}} B\right) \leq \Phi\left(A \sharp_{\frac{\alpha}{\beta}}\left(A \sigma_{f} B\right)\right),
$$

where $c_{g_{2}}>0$ is given by (2.8) with concave $g_{2}=g^{\alpha}$. 
Proof. By substituting $g_{1}(t)=g^{\beta}(t)$ and $g_{2}(t)=g^{\alpha}(t)$ for $t \in J$, we have

$$
g_{2} g_{1}^{-1}=(\cdot)^{\alpha} \circ g \circ g^{-1} \circ(\cdot)^{\frac{1}{\beta}}=(\cdot)^{\frac{\alpha}{\beta}},
$$

where the symbol $\circ$ stands for superposition. Thus $g_{2} g_{1}^{-1}(s)=s^{\frac{\alpha}{\beta}}, s \in g_{1}(J)$, is an operator monotone function. For this reason, Theorem 2.9, eq. (2.25), forces $(2.32)$.

Corollary 2.16. Let $f: J \rightarrow \mathbb{R}$ be a continuous real function with interval $J=$ $[m, M]$. Let $A$ and $B$ be $n \times n$ positive definite matrices such that $m A \leq B \leq M A$, $0<m<M \leq 1$.

If $\Phi: \mathbb{M}_{n}(\mathbb{C}) \rightarrow \mathbb{M}_{k}(\mathbb{C})$ is a strictly positive linear map and, $0<\alpha \leq \beta<1$,

$$
\begin{gathered}
t^{\beta} \leq f(t) \leq t^{\alpha} \quad \text { for } t \in J, \\
M^{\beta}=\max _{t \in J} f(t),
\end{gathered}
$$

then

$$
c_{g_{2}} \Phi(A) \sigma_{f} \Phi(B) \leq \Phi\left(A \sharp_{\alpha} B\right) \leq \Phi\left(A \sharp \frac{\alpha}{\beta}\left(A \sigma_{f} B\right)\right),
$$

where $c_{g_{2}}>0$ is given by (2.8) with $g_{2}(t)=t^{\alpha}$.

Proof. Employ Corollary 2.15 with $g(t)=t$.

We now apply Theorem 2.9 in the context of harmonic mean (cf. [6, Lemma 3.3]).

Corollary 2.17. Let $f: J \rightarrow \mathbb{R}$ and $g: J \rightarrow \mathbb{R}_{+}$be continuous real functions with intervals $J=[m, M]$ and invertible operator monotone $g$ on $J$. Let $A$ and $B$ be $n \times n$ positive definite matrices such that $m A \leq B \leq M A$.

If $\Phi: \mathbb{M}_{n}(\mathbb{C}) \rightarrow \mathbb{M}_{k}(\mathbb{C})$ is a strictly positive linear map and $0<\alpha \leq \beta<1$,

$$
\begin{gathered}
\left(\beta(g(t))^{-1}+1-\beta\right)^{-1} \leq f(t) \leq\left(\alpha(g(t))^{-1}+1-\alpha\right)^{-1} \quad \text { for } t \in J, \\
\max _{t \in J}\left(\beta(g(t))^{-1}+1-\beta\right)^{-1}=\max _{t \in J} f(t),
\end{gathered}
$$

then

$$
c_{g_{2}} \Phi(A) \sigma_{f} \Phi(B) \leq \Phi\left(A \sigma_{(\alpha(1 / g)+1-\alpha)^{-1}} B\right) \leq \Phi\left(A !_{\gamma}\left(A \sigma_{f} B\right)\right),
$$

where $\gamma=\frac{\alpha}{\beta}$ and $c_{g_{2}}>0$ is given by (2.8) with concave $g_{2}(t)=\left(\alpha(g(t))^{-1}+1-\right.$ $\alpha)^{-1}$.

Proof. By setting $g_{1}(t)=\left(\frac{\beta}{g(t)}+1-\beta\right)^{-1}$ and $g_{2}(t)=\left(\frac{\alpha}{g(t)}+1-\alpha\right)^{-1}$ for $t \in J$, we derive

$$
g_{2} g_{1}^{-1}(s)=\left[\frac{\alpha}{\beta} s^{-1}+\left((1-\alpha)-(1-\beta) \frac{\alpha}{\beta}\right)\right]^{-1} \quad \text { for } s \in g_{1}(J),
$$

with $\frac{\alpha}{\beta}+\left(1-\alpha-(1-\beta) \frac{\alpha}{\beta}\right)=1,0<\frac{\alpha}{\beta} \leq 1$ and $0 \leq 1-\alpha-(1-\beta) \frac{\alpha}{\beta}<1$. Therefore $g_{2} g_{1}^{-1}(s)=\left(\gamma s^{-1}+1-\gamma\right)^{-1}$ is an operator monotone function. So, in accordance with Theorem 2.9, inequality (2.26) implies (2.33). 
Corollary 2.18. Let $f: J \rightarrow \mathbb{R}$ be a continuous real function with interval $J=$ $[m, M]$. Let $A$ and $B$ be $n \times n$ positive definite matrices such that $m A \leq B \leq M A$, $0<m<M$.

If $\Phi: \mathbb{M}_{n}(\mathbb{C}) \rightarrow \mathbb{M}_{k}(\mathbb{C})$ is a strictly positive linear map and, for $0<\alpha \leq \beta<1$,

$$
\begin{gathered}
\left(\beta t^{-1}+1-\beta\right)^{-1} \leq f(t) \leq\left(\alpha t^{-1}+1-\alpha\right)^{-1} \quad \text { for } t \in J \\
\left(\beta M^{-1}+1-\beta\right)^{-1}=\max _{t \in J} f(t)
\end{gathered}
$$

then

$$
c_{g_{2}} \Phi(A) \sigma_{f} \Phi(B) \leq \Phi\left(A !_{\alpha} B\right) \leq \Phi\left(A !_{\gamma}\left(A \sigma_{f} B\right)\right),
$$

where $\gamma=\frac{\alpha}{\beta}$ and $c_{g_{2}}>0$ is given by $(2.8)$ with $g_{2}(t)=\left(\alpha t^{-1}+1-\alpha\right)^{-1}$.

Proof. Utilising Corollary 2.17 with $g(t)=t$ we get the desired result.

Acknowledgement. The author wishes to thank an anonymous referee for his helpful suggestions improving the readability of the paper.

\section{REFERENCES}

1. T. Ando, Concavity of certain maps on positive definite matrices and applications to Hadamard products, Linear Algebra Appl., 26 (1979), 203-241.

2. T. Ando, C.K. Li and R. Mathias, Geometric means, Linear Algebra Appl., 385 (2004), 305-334.

3. J. S. Aujla and H. L. Vasudeva, Inequalities involving Hadamard product and operator means, Math. Japon., 42 (1995), 265-272.

4. R. Bhatia, Matrix Analysis, Springer-Verlag, New York, 1997.

5. T. Furuta, J. Mićić, J. Pečarić and Y. Seo, Mond-Pečarić Method in Operator Inequalities, Element, Zagreb, 2005.

6. M. Ito, Y. Seo, T. Yamazaki and M. Tanagida, On a geometric property of positive definite matrices cone, Banach J. Math. Anal., 3 (2009), 64-76.

7. R. Kaur, M. Singh and J. S. Aujla, Generalized matrix version of reverse Hölder inequality, Linear Algebra Appl., 434 (2011), 636-640.

8. R. Kaur, M. Singh, J. S. Aujla and M. S. Moslehian, A general double inequality related to operator means and positive linear maps, Linear Algebra Appl., 437 (2012), 1016-1024.

9. F. Kubo, T. Ando, Means of positive linear operators, Math. Ann., 246 (1980), 205-224.

10. E.-Y. Lee, A matrix reverse Cauchy-Schwarz inequality, Linear Algebra Appl., 430 (2009), $805-810$.

11. J. Mičić, J. Pečarić and Y. Seo, Complementary inequalities to inequalities of Jensen and Ando based on the Mond-Pečarić method, Linear Algebra Appl., 318 (2000), 87-107.

12. M. Niezgoda, Accretive operators and Cassels inequality, Linear Algebra Appl., 433 (2010), $136-142$.

13. M. Niezgoda, Extensions of inequalities involving Kantorovich constant, Math. Inequal. Appl., 14 (2011), 935-946.

14. M. Niezgoda, On Diaz-Metcalf and Klamkin-McLenaghan type operator inequalities, J. Math. Inequal., 6 (2012), 289-297.

15. Y. Seo, Reverses of Ando's inequality for positive linear maps, Math. Inequal. Appl., 14 (2011), 905-910.

Department of Applied Mathematics and Computer Science, University of Life Sciences in Lublin, Akademicka 15, 20-950 Lublin, Poland.

E-mail address: marek.niezgoda@up.lublin.pl; bniezgoda@wp.pl 